\title{
Outcomes of Preserving the Hypertrophic Arachnoid Membrane in the Brain-meningioma Interface: Long-term Follow-up
}

\author{
Yoko NAKASU, ${ }^{1,2}$ Koichi MitsuYa, ${ }^{1}$ Satoshi NAKASU, ${ }^{3}$ Shoichi DEGUCHI, ${ }^{1}$ \\ and Nakamasa HAYASHI ${ }^{1}$
}

\author{
${ }^{1}$ Division of Neurosurgery, Shizuoka Cancer Center, Nagaizumi, Shizuoka, Japan \\ ${ }^{2}$ Department of Neurosurgery, Shiga University of Medical Science, Otsu, Shiga, Japan \\ ${ }^{3}$ Department of Neurooncology, Kusatsu General Hospital, Kusatsu, Shiga, Japan
}

\begin{abstract}
Abnormal hypertrophic arachnoid membranes are often observed in the brain-meningioma interface during microsurgery. They contain fibrosis and tumor cell clusters; however, preservation of the membranes does not always cause recurrence from the brain surface, and the optimal treatments in the interface remain unclear. We investigated the incidence of recurrence on the brain surface following extra-arachnoid dissection with an approach emphasizing preservation of the arachnoid membranes in meningiomas of World Health Organization (WHO) Grade I. The features of dissection cleavages in the interface were prospectively recorded at surgery. The patients were followed up with MR imaging regularly. In total, 111 patients were included. The median follow-up time was 97.0 (interquartile range [IQR] 70.0-124.0) months. The cleavages in the interface were classified into three subgroups: the Extra-H group $(n=56)$ with extraarachnoid resection and preservation of hypertrophic arachnoid membranes, the Extra- $\mathrm{N}$ group ( $n=39$ ) with extra-arachnoid resection having normal membranes, and the Subpial resection group $(n=16)$. Tumors recurred in $13(11.7 \%)$ patients at both the brain and dura mater $(n=1)$ or at the dura mater alone $(n=12)$. The median recurrence-free survival (RFS) of all recurrences was significantly related to the Simpson grades $(\mathrm{P}<\mathbf{0 . 0 1})$. For brain surface recurrence, the median RFS was not related to the subgroups. The Karnofsky Performance Scores (KPSs) significantly improved in the patients except for the Subpial group at 3 months after surgery. This study revealed that hypertrophic arachnoid membranes preserved on the brain surface rarely caused recurrence from the brain in WHO Grade I meningiomas after a long-term follow-up.
\end{abstract}

Keywords: arachnoid, oncology, pia mater, recurrence, resection

\section{Introduction}

Neurosurgeons have employed management strategies aimed at maximizing tumor control while minimizing neurological morbidity, especially for benign meningiomas. ${ }^{1-4)}$ Even after complete resection of convexity meningiomas, previous studies have reported consistent recurrence rates of $1.8-11.1 \%$ either from the brain or dural attachment. ${ }^{5-7)}$ Regarding surgical

Received July 6, 2021; Accepted September 17, 2021

Copyright $@ 2021$ The Japan Neurosurgical Society This work is licensed under a Creative Commons AttributionNonCommercial-NoDerivatives International License. factors related to recurrence and progression, discussions have been mainly focused on resection of the dura mater and bone. ${ }^{6-8,9,10)}$ Recurrence from the brain surface may relate to two factors, brain invasion of meningioma and tumor remnant in the arachnoid membranes over the brain surface. Only a few studies have explored the surgical techniques used at the interface. ${ }^{11-14)}$ Indeed, the clinical implications and optimal management of abnormal arachnoid membranes in the interface for preventing tumor recurrence and improving the patient neurological function remain unclear.

We previously reported that the connective tissue proliferation involves a spectrum of histological changes including hypertrophic arachnoid membranes 
and the so-called capsule composed of tumor stroma, arachnoid mater, and arachnoid trabecules. ${ }^{15)}$ The tumor cells were not highly proliferative in these arachnoid membranes with fibrotic changes. Therefore, we hypothesized that we could minimize surgical morbidity protecting the brain surface with arachnoid membranes in the interface while maximizing meningioma removal on the dural attachment. We prospectively recorded the features of the cleavage between the brain and the tumor during microsurgery for meningiomas, and followed the patients with immediate postoperative and regular magnetic resonance (MR) imaging. This study was conducted to investigate the incidence of tumor recurrence especially on the brain surface with extra-arachnoid and extrapial dissection preserving those hypertrophic arachnoid membranes, with a review of the long-term outcomes of patients with World Health Organization (WHO) Grade 1 meningiomas based on our 15 years of experience.

\section{Materials and Methods}

\section{Patient population}

After receiving approval from the institutional review board at Shizuoka Cancer Center (number 28-J168-28-1-3), we performed an observational study of patients with meningiomas treated at Shizuoka Cancer Center between 2003 and 2020. The study analyzed a consecutive series of 147 patients with intracranial meningiomas surgically treated with an approach emphasizing preservation of the arachnoid membranes on the brain by the senior neurosurgeon (YN) in the institute and followed for a minimum of 4 years. The patients underwent craniotomy for resection of a WHO Grade I meningioma as their initial therapy. We excluded patients with prior craniotomies for any reason, history of any other intracranial tumor, neurofibromatosis, or ventricular meningioma.

\section{Microsurgical technique and perioperative management}

The senior neurosurgeon prospectively employed a surgical strategy for meningiomas aimed at maximizing tumor control while minimizing the neurological deficits induced by surgery. Surgery was performed under general anesthesia with endotracheal intubation. Following small or medium standard craniotomy, resection was performed using classical microsurgical dissection techniques. Intratumoral volume reduction was performed, followed by dissection at the interface of the brain and tumor. Blunt or sharp dissection was conducted with special attention paid to the presence of the pial vascular supply, perforation of the pia mater, or hypertrophic arachnoid membrane. ${ }^{14)}$ Efforts were taken to preserve the arachnoid membranes over critical structures, even if this resulted in leaving small tumor remnants adhered to the structures. If the so-called tumor capsule of connective tissue proliferation over the brain was detected, we dissected it while leaving a layer and subarachnoid vessels over the brain surface. We performed subpial removal of the tumor in patients with firm adhesion to the brain surface through the perforated pia mater in non-eloquent areas, and the exposed brain surface was covered with oxidized cellulose and artificial cerebrospinal fluid.

Patients routinely underwent postoperative MR imaging with contrast within $48 \mathrm{~h}$ after surgery to confirm the extent of removal, and they underwent follow-up neurological examinations and MR imaging 3 and 6 months after surgery and annually thereafter.

\section{Data collection and classification of treatments in cleavage}

The features and dissection cleavages of the brain-meningioma interface were prospectively recorded by the consensus of the surgeons at surgery: condition of arachnoidal border (including critical tissue and vessels), dissection plane in the interface, preservation of the arachnoid membrane and pia mater, and extent of tumor resection in total and focused on dural attachment according to the Simpson grading system. ${ }^{8}$ The dissection plane and hypertrophic arachnoid membranes in the brain-tumor interface were classified into three subgroups: the Extra-H group, corresponding to extra-arachnoid and extrapial resection preserving layers of thick arachnoid membranes or capsule; the Extra-N group, corresponding to extra-arachnoid resection for cases with normal arachnoid membranes; and the Subpial group, corresponding to subpial total resection leaving no abnormal arachnoid membranes or capsule in the brain-tumor interface (Fig. 1, Supplementary Table 1. All Supplementary Tables are available Online.). Even a meningioma had a small pial defect in the interface, extraarachnoid resection was achieved leaving thick or normal arachnoid membranes in Extra- $\mathrm{H}$ and Extra-N groups.

In this study, data collected included the patient age at surgery, sex, preoperative and postoperative functional status, tumor size, surrounding brain edema, radiological recurrence date, and WHO grades (classification in 2016). ${ }^{16)}$ The tumor size was measured by the maximum diameter on contrastenhanced MR images. The extent of removal was confirmed by MR imaging within 48 hours after 


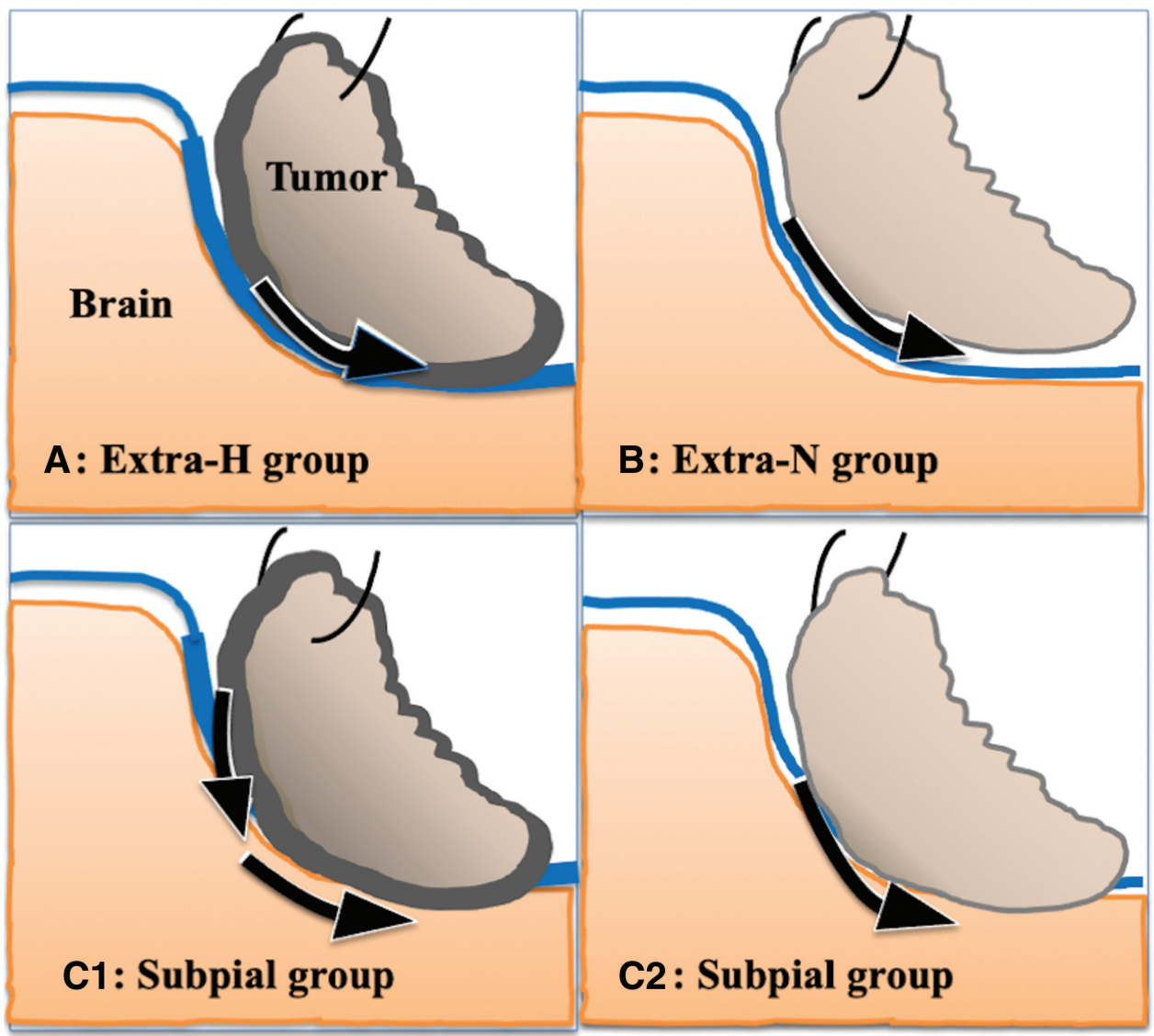

Fig. 1 Schematic drawings of subgroups showing surgical cleavage (black arrows) in the brain-meningioma interface. (A) Extra-H group: Extra-arachnoid dissection cleavage is made between a meningioma with fibrotic change (gray) and a hypertrophic arachnoid membrane (thick blue line). (B) Extra-N group: Extra-arachnoid cleavage is made between a meningioma and an arachnoid membrane (thin blue line) without hypertrophic changes. C1 and C2, Subpial group: Subpial dissection for a meningioma with fibrotic change (gray) merged with a hypertrophic arachnoid membrane (thick blue line) and pia mater (thin orange line) (C1) and for another with a disrupted arachnoid membrane (thin blue line) and pia mater (thin orange line) without fibrotic changes (C2).

surgery in each case. Neurological morbidity was defined as aggravation of preoperative neurological deficits or new deficits that persisted beyond the first 30 days after surgery. The performance status was assessed immediately before surgery and 3 months postoperatively using the Karnofsky Performance Score (KPS). Any death within 30 days postoperatively was considered an operative death.

The preoperative evaluation of all patients included T1- and T2-weighted MR imaging with and without contrast media. Preoperative embolization was performed for larger tumors. Recurrence or progression was defined as documented significant growth of the tumor on follow-up MR images with consensus at our neuroradiology board. No patients underwent immediate postoperative irradiation for residual disease.

\section{Statistical analyses}

The analysis was conducted using the $\mathrm{R}$ software program, version 3.5.1. Recurrence was analyzed using the Kaplan-Meier method. The log-rank test was used to assess the significance of recurrence rates. The Cox proportional hazard test was performed for multivariate analyses of recurrence. The nonparametric Kruskal-Wallis model was used to compare independent significant factors of recurrence time. Post hoc tests of continuous variables were performed by the Steel-Dwass test. Fisher's test was also used in the analysis of categorical parameters. Postoperative KPS changes were evaluated using Wilcoxon signed rank test. Continuous variables, including the patient age, tumor size, time to recurrence and duration of follow-up, were expressed as the median 
values with the interquartile range (IQR). A p value of $<0.05$ was considered significant.

\section{Results}

\section{Patients' characteristics and operative data}

A total of 111 patients were enrolled (Supplementary Table 2). Eighty-one were women. The mean age at surgery was $59.8 \pm 12.9$ years old. Tumor sizes ranged from 10 to $104 \mathrm{~mm}$ (median 31 [25-44] mm). There were 70 skull base meningiomas (63\%) and 41 non-skull base meningiomas (36.9\%). This high ratio of skull base lesions was due to the tendency of patients to be referred to us in our local district. Ninety-eight $(88.3 \%)$ patients received Simpson Grade I to III resection and $13(11.7 \%)$ Grade IV resection. The median duration of postoperative follow-up was 97.0 [70.0-124.0] months.

Dissection cleavages in the interface were extrapial for 95 patients (85.6\%; Extra-H and Extra-N groups) and subpial for 16 patients (14.4\%; Subpial group). Of the 95 meningiomas with extrapial dissection, 56 had hypertrophic arachnoid membranes that were intently preserved in the interface (Extra- $\mathrm{H}$ group). Table 1 shows the differences in the clinical features of the subgroups. The patients in the Extra- $\mathrm{H}$ group were older, and those in the Subpial group had significantly larger meningiomas with a higher incidence of perifocal edema, pial feeders, and pial disruption than other groups. There were small pial defects less than one-quarter of the total interface observed in patients of the Extra-H $(28 \%)$ and Extra-N (20.5\%) groups. Immediate postoperative MR imaging demonstrated enhancement of hypertrophic arachnoid membrane in $66 \%$ of patients from Extra-H group. Figure 2 demonstrates preoperative and postoperative MR images with contrast media in representative patients of the Extra- $\mathrm{H}$ group.

\section{Recurrence of meningiomas}

Tumor recurrence or progression was radiologically detected in $13(11.7 \%)$ patients: 1 at both the brain and dura mater, 12 only at the dura mater (Fig. 3A). Table 2 shows the results of uni- and multivariate analyses. The analysis of the 13 total recurrences did not show significant differences in the regrowth or the recurrence-free survival (RFS) among subgroups (log-rank $\mathrm{P}=0.68$, Fig. 3B). However, the difference reached statistical significance among Simpson grades (log-rank $\mathrm{P}<0.001$, multivariate hazard ratio [HR]: 2.18; $\mathrm{P}=0.016$, Fig. 3C).

Tumors recurred at the brain surface in a single $(0.9 \%)$ patient (Fig. 2B) associated with recurrence in the dura mater at the same time. Only a univariate analysis was performed for factors in the recurrence in the brain, as the number of events was too small to run calculations to converge for a multivariate analysis. The differences in the RFS from the brain surface did not reach significance for the gender, age, tumor size, location, preoperative KPS, Simpson grade, or interface subgroup (Table 2).

\section{Morbidity and functional status by surgical treatments of the interface}

There were no surgical mortalities. Two patients experienced postoperative transient neurological

Table 1 Differences in patient features among the subgroups

\begin{tabular}{|c|c|c|c|c|}
\hline Factors & $\begin{array}{c}\text { Extra-H Group N = } 56 \\
\text { Figure 1A }\end{array}$ & $\begin{array}{c}\text { Extra-N Group N = } 39 \\
\text { Figure 1B }\end{array}$ & $\begin{array}{c}\text { Subpial Group } N=16 \\
\text { Figure } 1 \mathrm{C} 1 / 2\end{array}$ & $\begin{array}{c}\text { Kruskal-Wallis } \\
\text { analysis }\end{array}$ \\
\hline Size $(\mathrm{mm})$ mean/SD & $37.7 / 16.6$ & $28.4 / 11.8$ & $42.6 / 15.8$ & $\mathrm{P}<0.001$ \\
\hline Post hoc analysis & \multicolumn{3}{|c|}{$\mathrm{H}: \mathrm{N} P=0.009, \mathrm{~N}:$ Sub $\mathrm{P}=0.004$, Sub:H P $=0.45$} & \\
\hline Age (years) mean/SD & $63.5 / 11.5$ & $54.8 / 12.7$ & $58.8 / 14.4$ & $\mathrm{P}=0.01$ \\
\hline Post hoc analysis & \multicolumn{3}{|c|}{$\mathrm{H}: \mathrm{N} P=0.006, \mathrm{~N}: S u b \mathrm{P}=0.55$, Sub:H P $=0.55$} & \\
\hline Location SB:non-SB & $35: 21$ & 29:10 & $6: 10$ & $P=0.039$ \\
\hline Perifocal edema Y:N & $25: 31$ & $9: 30$ & $12: 4$ & $\mathrm{P}=0.0014$ \\
\hline Pial feeders Y:N & $12: 44$ & $5: 34$ & $6: 10$ & $\mathrm{P}=0.13$ \\
\hline Pia disruption Y:N & $16: 40$ & $8: 31$ & $14: 2$ & $\mathrm{P}<0.001$ \\
\hline Simpson Gr I-III:IV & $52: 4$ & $31: 8$ & $15: 1$ & $\mathrm{P}=0.256$ \\
\hline KPS mean/SD Preop & $84.6 / 15.5$ & $90.3 / 12.2$ & $90.6 / 10.0$ & \\
\hline Postop & $91.3 / 10.3$ & $96.2 / 5.9$ & $90.6 / 16.9$ & \\
\hline Wilcoxon signed rank test & $\mathrm{P}<0.001$ & $\mathrm{P}<0.001$ & $\mathrm{P}=0.75$ & \\
\hline
\end{tabular}

Preop: preoperative, Postop: postoperative, SB: skull base, SD: standard deviation, Y: N: Yes or No. 

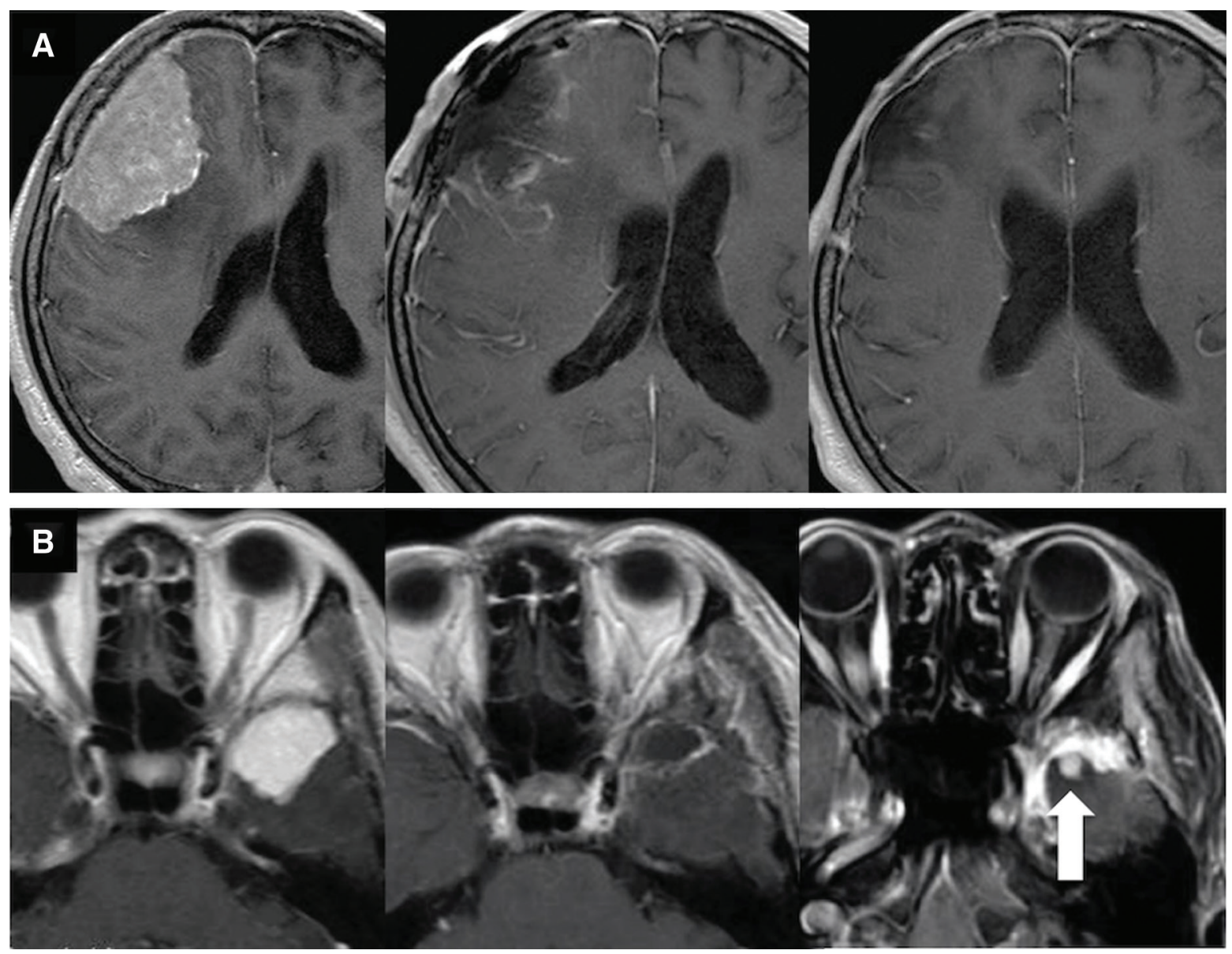

Fig. 2 MR images showing preoperative meningioma and early postoperative enhancement at the brain surface. (A) Axial enhanced T1-weighted images demonstrating a preoperative tumor (left), redundant sheets of hypertrophic arachnoid membranes on the brain surface 17 hours after resection (middle), and disappearance of the abnormal enhancement 6 months after surgery (right) for a 60-mm meningioma by Simpson Grade II and Extra-H resection. The patient has been asymptomatic without recurrence 84 months after surgery. (B) Axial enhanced T1-weighted images demonstrating a preoperative tumor (left), sheets of hypertrophic arachnoid membranes on the brain surface 20 hours after resection (middle), and recurrent lesions at the brain surface (white arrow) and dura mater 60 months after initial surgery for a $30-\mathrm{mm}$ meningioma by Simpson Grade II and Extra-H resection (right). MR: magnetic resonance.

deficits but had recovered to their preoperative condition by the 3-month follow-up visit after surgery. Another three in the Subpial group had permanent worsening of their function. These three patients accounted for $2.7 \%$ of all patients and $18.8 \%$ of those in the Subpial group.

Preoperatively, 12 patients were moderately disabled with a KPS $<70 / 100$. The preoperative KPS was significantly lower in patients $\geq 60$ than in those $<60$ years old $(\mathrm{P}=0.001)$. The functional score by KPS was significantly improved in Extra-H and Extra-N groups, but not in the Subpial group at 3 months after surgery (Fig. 3D).
A preoperative history of epilepsy was noted in six patients, two of whom had long histories of idiopathic epilepsy treated at an epilepsy center. Postoperatively, novel epileptic seizures developed in two patients, including one patient at 6 months after total and Extra-H resection for a parietal convexity meningioma and another at 12 months after subpial total removal (Subpial group) for a frontal convexity meningioma. At the latest follow-up visits, 14 patients, including these two patients, had received anticonvulsants and were all seizure-free. The rate of anticonvulsant prescription showed no significant difference among the subgroups $(\mathrm{P}=0.26)$. 


\section{A}

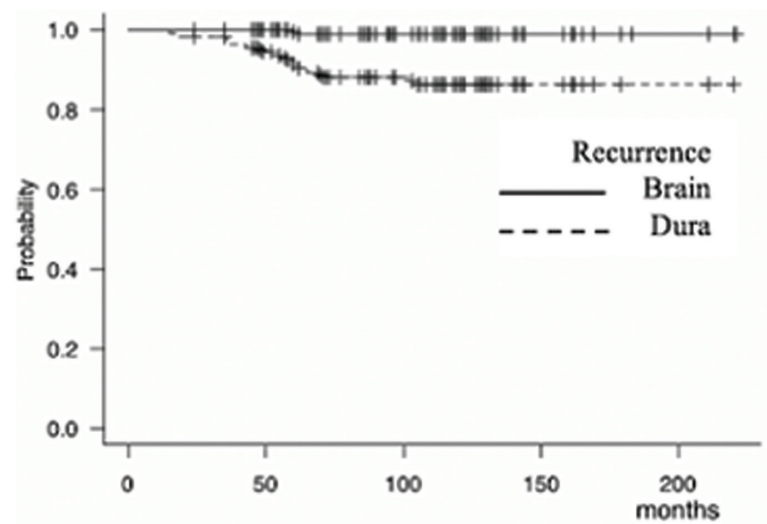

Number at risk

$\begin{array}{cl}\text { Brain } 111 & 99 \\ \text { Dura } 111 & 94\end{array}$

C

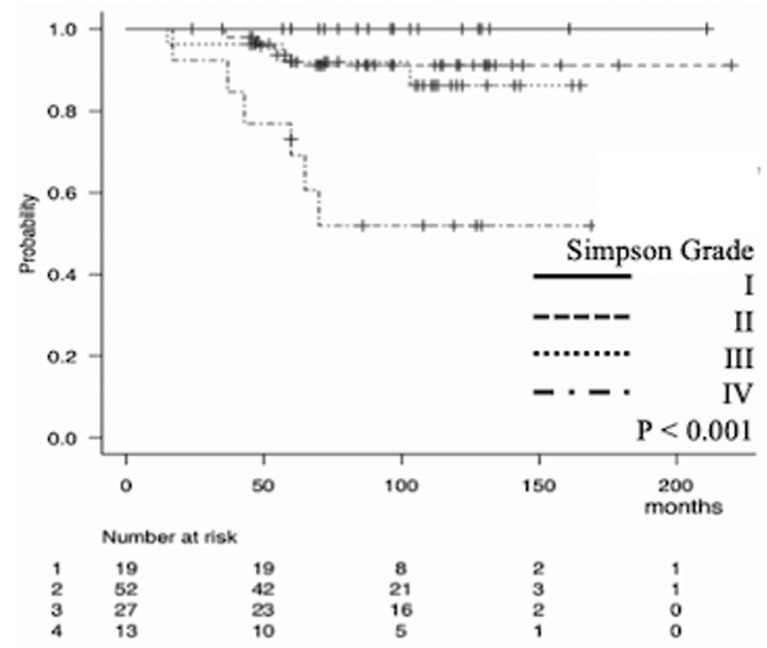

B

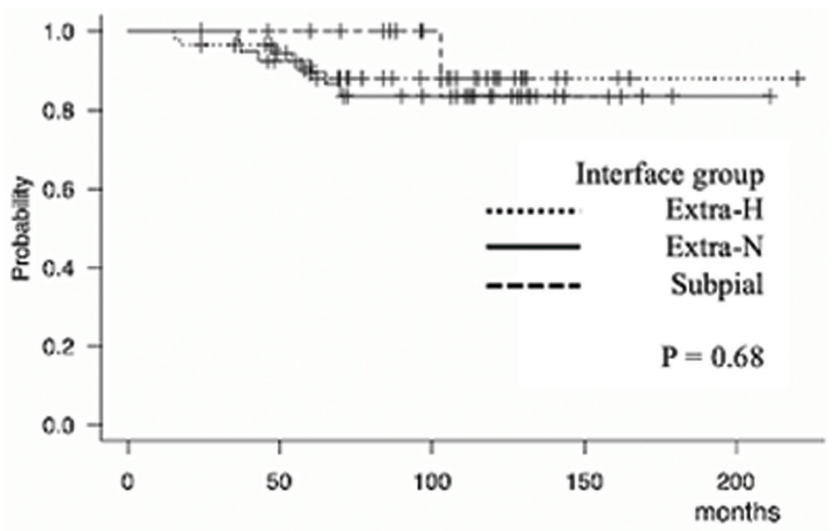

Number at risk

$\begin{array}{rrrcrl}\text { Ext-N } & 39 & 34 & 21 & 3 & 1 \\ \text { Subpial } & 16 & 14 & 6 & 2 & 0 \\ \text { Ext-H } & 56 & 46 & 23 & 3 & 1\end{array}$

D

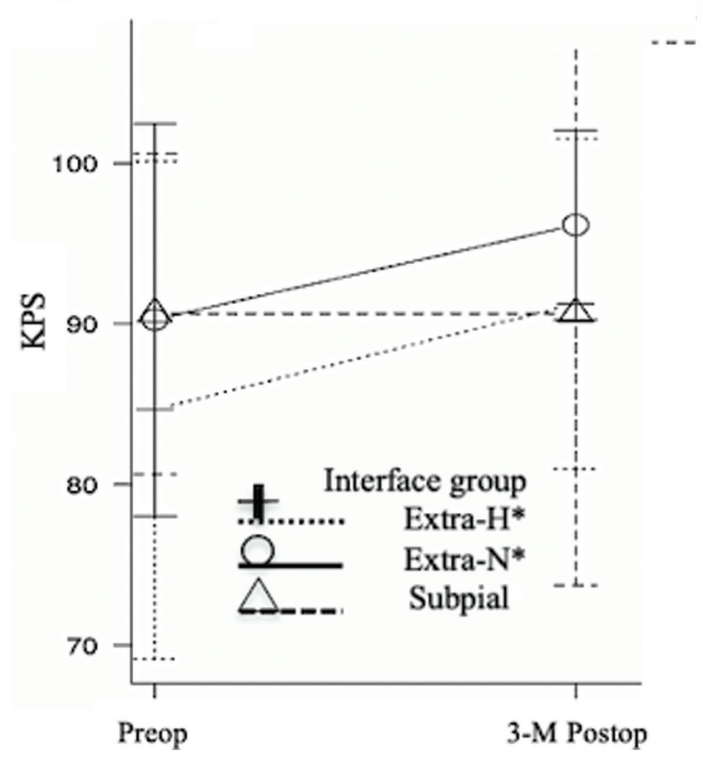

Fig. 3 (A-C) Kaplan-Meier curves of the actuarial RFS probability according to the sites at the brain surface and dura mater (A), according to the interface subgroups (B), and according to the Simpson grades (C). (D) Plots of the means and standard deviations for the preoperative and 3-month postoperative KPSs. Patients from the Extra-H group and Extra-N group showed significant improvements in their postoperative scores, but patients from the Subpial group remained ( ${ }^{*}$ Wilcoxon signed rank test; $\left.\mathbf{P}<0.01\right)$. KPS: Karnofsky Performance Scores, RFS: recurrence-free survival.

\section{Discussion}

Abnormal arachnoid membrane at the interface of the brain and meningioma

The microscopic anatomy of the interface revealed that meningiomas induce fibrotic changes and abnormal arachnoid membranes of varying thickness and disruption with or without hypertrophic changes. ${ }^{15)}$ These abnormal changes of arachnoid membranes seem mixed, redundant or partial in the interface. In this series of 111 consecutive patients, hypertrophic arachnoid membranes were found and preserved in the interface of 56 (50.5\%) patients (Extra-H group), and hypertrophic and/or disrupted arachnoid membranes were removed in $16(14.4 \%)$ patients (Subpial group); therefore, abnormal arachnoid membranes were found in 72 patients $(64.9 \%)$ during microsurgery. Sindou 
Table 2 Uni- and multivariate analyses of the RFS

\begin{tabular}{|c|c|c|c|}
\hline \multirow{2}{*}{ Variable } & \multicolumn{2}{|c|}{ All recurrence $(\mathrm{N}=13)$} & \multirow{2}{*}{$\begin{array}{c}\text { Recurrence in the } \\
\text { brain }(\mathrm{N}=1) \\
\begin{array}{c}\text { Univariate analysis } \\
\text { (Log-rank) }\end{array}\end{array}$} \\
\hline & $\begin{array}{l}\text { Univariate analysis } \\
\text { (Log-rank) }\end{array}$ & $\begin{array}{l}\text { Multivariate } \\
\text { analysis (Cox) }\end{array}$ & \\
\hline Age (>60 years) & $\mathrm{P}=0.035$ & $\mathrm{HR} 2.79 \mathrm{P}=0.13$ & $\mathrm{P}=0.32$ \\
\hline Sex & $P=0.85$ & & $P=0.53$ \\
\hline Size (>30 mm) & $\mathrm{P}=0.31$ & HR $0.77 \mathrm{P}=0.65$ & $P=0.26$ \\
\hline Location (SB:NSB) & $P=0.058$ & HR $2.37 \mathrm{P}=0.29$ & $P=0.40$ \\
\hline KPS (>70/100) & $\mathrm{P}=0.47$ & & $P=0.66$ \\
\hline Brain edema & $\mathrm{P}=0.75$ & & $\mathrm{P}=0.24$ \\
\hline Pial feeders & $\mathrm{P}=0.97$ & & $\mathrm{P}=0.075$ \\
\hline Subgroup by interface & $P=0.68$ & & $P=0.55$ \\
\hline Simpson grade & $\mathrm{P}<0.001$ & HR $2.18 \mathrm{P}=0.016$ & $P=0.73$ \\
\hline
\end{tabular}

HR: hazard ratio, KPS: Karnofsky performance score, NSB: non-skull base, RFS: recurrence-free survival, SB: skull base.

and Alaywan reported that the arachnoid cleavage plane was achieved in only $54.6 \%$ of 150 meningiomas. ${ }^{11)}$ Subsequently, Alvernia et al. reported their experience in surgical cleavability restricted to convexity meningiomas of WHO Grade . $^{12)}$ In those studies, subpial dissection was discussed without any information on abnormal arachnoid membranes at the interface. Kamitani et al. first reported microscopic tumor cell clusters in thick arachnoid membranes surrounding meningioma and assumed their clinical importance in recurrence ${ }^{13)}$ On the other hand, the incidence of arachnoid disruption (perforation) was reported in two studies to be $26 \%$ and $63.6 \%{ }^{17,18)}$ The arachnoid and pial disruption found during surgical dissection may be induced by several mechanisms: by tumor protrusion on the brain surface together with a reduced consistency of pia-arachnoid membranes or by true brain invasion. True brain invasion is a pathological diagnosis defined as tumor tissue within the adjacent brain without a separating connective tissue layer. ${ }^{19,20)}$ Therefore, surgeons cannot differentiate true brain invasion from tumor protrusion into the cortex through the simple perforation of pia-arachnoid membranes when they take an approach emphasizing preservation of the arachnoid membranes on the brain.

\section{Abnormal arachnoid membranes and recurrence}

The precise recurrence sites, whether the dura mater or brain surface, have been rarely reported even in articles discussing the surgical cleavage or surgical treatment at the brain-tumor interface.
Kamitani et al. reported that thick arachnoid membrane remnants were related to meningioma recurrence; however, the details concerning the recurrence sites were unclear. ${ }^{13)}$ Yamasaki et al. reported meningioma recurrence in 6 of 54 patients after Simpson Grade I resection of convexity meningiomas, and they were all found in the brain. ${ }^{5)}$ The authors presented no findings at the brain-tumor interface or dissection cleavage in their series. Several studies have reported that subpial cleavage and brain invasion showed predictive value for recurrence after surgery in cases of intracranial meningiomas. ${ }^{11,12,19)}$ However, the exact focuses of recurrence, possible remnants, and abnormal membranes on the brain surface were not clearly presented in these studies. The features of recurrence at the brain surface may be elucidated by three-dimensional MR imaging, which is now widely used for patients with meningioma.

The present study revealed only one recurrence in the brain among 56 patients in the Extra- $\mathrm{H}$ group after a median follow-up of 97 months (Table 2, Fig. 3C). The result suggests that remnant meningioma cells in the preserved hypertrophic arachnoid membranes might become the focus of rare tumor recurrence after gross total removal of the tumor body. Extra-H group patients were significantly older than those in the Extra-N group or Subpial group (Table 1). Therefore, conservative dissection at the interface should be seriously considered, as stripping the abnormal membranes from the brain surface might cause neurological deterioration or sequelae, especially in older patients. 
In addition, the present study showed the recurrence rate in the dura mater was $11.7 \%$ with a median follow-up of 97 months, which is within the range reported in the literature. ${ }^{16,21,22)}$ And the study revealed that recurrence at the dura mater was not related to the interface subgroups (Table 2 , Fig. 3B). These results suggest that the presence of hypertrophic arachnoid membrane alone does not reflect a high or low proliferation potential of meningioma recurrence in the dura.

\section{Operative morbidity and treatments of abnormal arachnoid membranes}

In our series, there were no surgical mortalities. The overall surgical morbidity rate was $4.5 \%$ (5 in 111 patients), including transient palsy. Previous studies have reported that the rate of experiencing a new or worsened deficit ranged from $8.3 \%$ to $14.8 \% .{ }^{11,23)}$ In this study, 4 of 5 patients who suffered neurological deterioration were in the Subpial group, which showed a morbidity rate of $25 \%$ (4/16). Furthermore, the postoperative KPS did not improve in the Subpial group while those significantly improved in the other groups (Table 1, Fig. 3D). All patients who had a deteriorated in KPS in the Subpial group were $\geq 60$ years old. These operative morbidity factors need to be assessed in addition to the tumor location and comorbidity that have been discussed in the literature. ${ }^{23)}$

The onset of seizures after meningioma surgery is a concern, especially after subpial resection. In the present study, new-onset seizures occurred in two patients $(1.8 \%$; one in the Extra-H group and another in the Subpial group). This result is in line with recent literature indicating new-onset seizure rates of $1.9 \%-19.4 \%{ }^{23,24)}$ All 111 of the present patients were seizure-free at their last follow-up visit, and $12.6 \%$ were under control with anticonvulsants.

\section{Study limitations}

The observational nature of the study along with the subjective and qualitative assessments of the surgical findings without random assignment needs to be considered when evaluating the results. Thorough sampling of the hypertrophic arachnoid membrane for pathological examination was not allowed to preserve the brain and critical structures. Immediate postoperative MR imaging did not always demonstrate enhancement of the preserved membranes, making volumetric assessment of the remnants impossible on the brain surface after surgery. Recurrence was not confirmed histologically in several cases, as the recurrent tumors were diagnosed at a small size in asymptomatic patients by routine follow-up imaging.

Intraoperative decision of dissection cleavage is difficult for surgeons especially over critical structures if an intraoperative frozen section suggested that the tumor had borderline aggressive features. During the study period, we removed nine WHO Grade II meningiomas with the same approach of preserving the arachnoid membranes in the interface. Two $(22.2 \%)$ of nine WHO Grade II meningiomas recurred at the brain surface, whereas one $(0.9 \%)$ of 111 WHO Grade I meningiomas (log-rank P <0.0001). Careful follow-up with MR imaging should be planned for patients who undergo membranepreserving resection at the interface. We believe that the inclusion of more patients and extended follow-up would aid in validating our findings.

\section{Conclusions}

The surgical preservation of hypertrophic arachnoid membranes in the brain-tumor interface did not increase the risk of recurrence from the brain surface in WHO Grade I meningiomas. The postoperative KPS did not improve in patients who received subpial resection. Considering the postoperative quality of life in meningioma patients, the authors believe that aggressive attempts to remove abnormal arachnoid membranes in the interface are less beneficial than simple resection for WHO Grade I meningiomas. However, careful follow-up with MR imaging should be planned for patients who undergo membrane-preserving resection at the interface.

\section{Acknowledgments}

We would like to thank Dr. Sayaka Ito (Nagahama Red Cross Hospital, Shiga, Japan) and Dr. Ayako Shima (Koto Memorial Hospital, Shiga, Japan) for performing the patient follow-up, and Mr. Brian Quinn (Japan Medical Communication) for editing a draft of this manuscript.

\section{Conflicts of Interest Disclosure}

The authors have no conflict of interest to declare.

All authors have registered online self-reported COI Disclosure Statement Forms for JNS members.

\section{References}

1) Raffa G, Picht T, Scibilia A, et al.: Surgical treatment of meningiomas located in the rolandic area: the role of navigated transcranial magnetic stimulation for preoperative planning, surgical strategy, and 
prediction of arachnoidal cleavage and motor outcome. J Neurosurg 133: 107-118, 2020

2) Gozal YM, Alzhrani G, Abou-Al-Shaar H, Azab MA, Walsh MT, Couldwell WT: Outcomes of decompressive surgery for cavernous sinus meningiomas: long-term follow-up in 50 patients. J Neurosurg 132: $380-387,2020$

3) Kim JW, Jung HW, Kim YH, et al.: Petroclival meningiomas: long-term outcomes of multimodal treatments and management strategies based on 30 years of experience at a single institution. J Neurosurg 132: 1675-1682, 2020

4) Nakasu S, Nakasu Y: Natural history of meningiomas: review with meta-analyses. Neurol Med Chir (Tokyo) 60: 109-120, 2020

5) Yamasaki F, Yoshioka H, Hama S, Sugiyama K, Arita K, Kurisu K: Recurrence of meningiomas. Cancer 89: 1102-1110, 2000

6) Morokoff AP, Zauberman J, Black PM: Surgery for convexity meningiomas. Neurosurgery 63: 427-433; discussion 433-434, 2008

7) Hasseleid BF, Meling TR, Rønning P, Scheie D, Helseth E: Surgery for convexity meningioma: Simpson Grade I resection as the goal: clinical article. J Neurosurg 117: 999-1006, 2012

8) SIMPSON D: The recurrence of intracranial meningiomas after surgical treatment. J Neurol Neurosurg Psychiatry 20: 22-39, 1957

9) Sughrue ME, Kane AJ, Shangari G, et al.: The relevance of Simpson Grade I and II resection in modern neurosurgical treatment of World Health Organization Grade I meningiomas. J Neurosurg 113: 1029-1035, 2010

10) Gousias K, Schramm J, Simon M: The Simpson grading revisited: aggressive surgery and its place in modern meningioma management. J Neurosurg 125: 551-560, 2016

11) Sindou MP, Alaywan M: Most intracranial meningiomas are not cleavable tumors: anatomic-surgical evidence and angiographic predictibility. Neurosurgery 42: 476-480, 1998

12) Alvernia JE, Dang ND, Sindou MP: Convexity meningiomas: study of recurrence factors with special emphasis on the cleavage plane in a series of 100 consecutive patients. J Neurosurg 115: 491-498, 2011

13) Kamitani H, Masuzawa H, Kanazawa I, Kubo T: Recurrence of convexity meningiomas: tumor cells in the arachnoid membrane. Surg Neurol 56: 228-235, 2001

14) Rai SKR, Mancarella C, Goel AH: Brain tumor interface dissection technique with surgical blade from laboratory to neurosurgical operating room. World Neurosurg 100: 601-606, 2017

15) Nakasu S, Fukami T, Jito J, Matsuda M: Microscopic anatomy of the brain-meningioma interface. Brain Tumor Pathol 22: 53-57, 2005

16) Perry A, Rushing EJ, Louis DN, et al.: Meningioma. In: Louis DN, Ohgaki H, Wiestler OD, Cavenee WK (eds): WHO Classification of Tumours of the Central Nervous System, revised ed 4. Lyon International Agency for Research on Cancer, 2016, pp 232-245

17) Takahashi JA, Ueba T, Hashimoto N, Nakashima Y, Katsuki N: The combination of mitotic and Ki-67 indices as a useful method for predicting short-term recurrence of meningiomas. Surg Neurol 61: 149-155; discussion 155-156, 2004

18) Pfisterer WK, Hendricks WP, Scheck AC, et al.: Fluorescent in situ hybridization and ex vivo $1 \mathrm{H}$ magnetic resonance spectroscopic examinations of meningioma tumor tissue: is it possible to identify a clinically-aggressive subset of benign meningiomas? Neurosurgery 61: 1048-1059; discussion 1060-1061, 2007

19) Perry A, Scheithauer BW, Stafford SL, Lohse CM, Wollan PC: "Malignancy" in meningiomas: a clinicopathologic study of 116 patients, with grading implications. Cancer 85: 2046-2056, 1999

20) Nakasu S, Nakasu Y: Prognostic significance of brain invasion in meningiomas: systematic review and meta-analysis. Brain Tumor Pathol 38: 81-95, 2021

21) Voß KM, Spille DC, Sauerland C, et al.: The Simpson grading in meningioma surgery: does the tumor location influence the prognostic value? J Neurooncol 133: 641-651, 2017

22) Zhu Z, Wang $\mathrm{C}$, Xu J, et al.: A quantified risk-scoring system for the recurrence of meningiomas: results from a retrospective study of 392 patients. Front Oncol 10: 585313, 2020

23) Corell A, Thurin E, Skoglund T, et al.: Neurosurgical treatment and outcome patterns of meningioma in Sweden: a nationwide registry-based study. Acta Neurochir (Wien) 161: 333-341, 2019

24) Gupte TP, Li C, Jin L, et al.: Clinical and genomic factors associated with seizures in meningiomas. J Neurosurg doi.org/10.3171/2020.7.JNS201042 Epub 2020 Dec 4

Corresponding author: Yoko Nakasu, MD, PhD Department of Neurosurgery, Shiga University of Medical Science, Setatsukinowa, Otsu, Shiga 520-2192, Japan. e-mail:nakasu@belle.shiga-med.ac.jp 